\title{
Le bibliothécaire québécois en entreprise : le défi du changement
}

\section{The corporate librarian in Quebec: the challenge of change El bibliotecario quebequense en el medio empresarial: el veto del cambio}

\author{
Lucie Dion, Robert Galipeau, Émilie Gagnon et Emily Toms
}

Volume 54, numéro 2, avril-juin 2008

Topographie du Québec documentaire

URI : https://id.erudit.org/iderudit/1029324ar

DOI : https://doi.org/10.7202/1029324ar

Aller au sommaire du numéro

Éditeur(s)

Association pour l'avancement des sciences et des techniques de la

documentation (ASTED)

ISSN

0315-2340 (imprimé)

2291-8949 (numérique)

Découvrir la revue

Citer cet article

Dion, L., Galipeau, R., Gagnon, É. \& Toms, E. (2008). Le bibliothécaire québécois en entreprise : le défi du changement. Documentation et bibliothèques, 54(2),

143-146. https://doi.org/10.7202/1029324ar
Résumé de l'article

Cet article trace le portrait des spécialistes de l'information et des bibliothèques spécialisées implantées dans les entreprises au Québec. Nous décrirons comment les bibliothécaires réagissent aux défis posés par la mondialisation, la centralisation des ressources informationnelles, l'apparition des centres d'information et des services virtuels, l'optimisation de l'utilisation des technologies de l'information, l'intégration de la gestion de l'information, le marketing des services au sein de l'organisation, la gestion des connaissances, l'apparition d'usagers toujours plus compétents et, finalement, l'obtention de la reconnaissance professionnelle.
Tous droits réservés (C) Association pour l'avancement des sciences et des techniques de la documentation (ASTED) et Corporation des bibliothécaires professionnels du Québec (CBPQ), 2008
Ce document est protégé par la loi sur le droit d'auteur. L'utilisation des services d’Érudit (y compris la reproduction) est assujettie à sa politique d'utilisation que vous pouvez consulter en ligne. 


\section{Le bibliothécaire québécois en entreprise : le défi du changement}

\author{
LUCIE DION \\ Directrice, Programme d'Investissements \\ Communautaires \\ Précédemment Directrice, \\ Centre d'information d'affaires Alcan \\ lucie.dion@alcan.com
}

\section{ROBERT GALIPEAU}

Directeur, Centre d'information d'affaires Rio

Tinto Alcan (Montréal)

robert.galipeau@alcan.com

\author{
ÉMILIE GAGNON \\ Spécialiste de l'information, \\ Recherche et veille \\ Centre d'information d'affaires Rio Tinto Alcan \\ emilie.gagnon@alcan.com
}

\section{EMILY TOMS}

Spécialiste de l'information,

Recherche et veille

Centre d'information d'affaires Rio Tinto Alcan emily.toms@alcan.com

\section{RÉSUMÉ | ABSTRACTS | RESUMEN}

Cet article trace le portrait des spécialistes de l'information et des bibliothèques spécialisées implantées dans lẹs entreprises au Québec. Nous décrirons comment les bibliothécaires réagissent aux défis posés par la mondialisation, la centralisation des ressources informationnelles, l'apparition des centres d'information et des services virtuels, l'optimisation de l'utilisation des technologies de l'infórmation, l'intégration de la gestion de l'information, le marketing'des services au sein de l'organisation, la gestion des connaissances, l'apparition d'usagers toujours plus compétents et, finalement, l'obtention de la reconnaissance professionnelle.

The corporate librarian in Quebec: the challenge of change This article provides an overview of information specialists working within corporate libraries in Quebec. We will describe how librarians are reacting to challenges such as globalization, the centralization of information resources, the advent of virtual information and service centres, information technology use and optimization, integration of information management practices, marketing of services within an organization, records management, an increasingly competent workforce, and, finally, professional recognition.

El bibliotecario quebequense en el medio empresarial : el reto del cambio

El presente artículo presenta un retrato de los especialistas de la información y de las bibliotecas especializadas que se han ido implantando en el medio empresarial en Québec. En él describiremos las reacciones de los bibliotecarios que trabajan en empresas a los retos impuestos por la globalización, la concentración de los recursos informacionales, la aparición de centros virtuales de información y de servicios, la optimización del uso de las tecnologías de la información, la integración de la gestión de la información, la promoción de los servicios dentro de la empresá, la gestión del conocimiento, la aparición de usuarios cada vez más competentes $y$, finalmente, la obtención del reconocimiento profesional.
$\mathrm{D}$ ANS LA PRÉSENTATION du numéro thématique de Documentation et bibliothèques sur les enjeux et les défis de la bibliothèque spécialisée paru en 1997, il est mentionné que le centre d'information spécialisé « (...) se définit à l'image des besoins de ses usagers. Chaque bibliothèque spécialisée trouve des solutions différentes et innovatrices aux enjeux et défis qu'elle affronte ${ }^{1}$. » Ce constat s'applique encore, dix ans plus tard, et cet état des lieux s'en veut le portrait. Le présent article décrit la réalité des centres d'information dans les entreprises ou les organisations parapubliques québécoises œuvrant dans les domaines financier et commercial uniquement. Dans un premier temps, les auteurs présentent les enjeux et défis actuels auxquels sont confrontées les bibliothèques spécialisées en entreprise pour ensuite dresser un portrait statistique et décrire l'organisation, les clientèles desservies et les services offerts. Ils terminent cet état des lieux en dégageant les tendances et les enjeux de ce milieu particulier.

\section{Les enjeux et les défis}

Les entreprises québécoises n’ont pas échappé ces dernières années au phénomène de la mondialisation ni à celui des fusions et acquisitions. Mentionnons, à titre d'exemples, l'achat d'Alcan Inc. par la firme angloaustralienne Rio Tinto et l'offre d'achat de Teachers à Bell Canada en 2007. Ces enjeux économiques entraînent nécessairement des conséquences sur l'organisation des entreprises touchées et sur celle des centres d'information. La centralisation de la gestion des ressources documentaires en un seul service d'information mondial ou national est une réponse typique à cette réalité. On voit aussi de plus en plus la mise en commun et en réseau des centres d'information dans les organisations afin de mieux gérer les ressources et offrir un service optimal. Ainsi, dans un cabinet d'avocats

1. Dion, Lucie "Enjeux et défis de la bibliothèque spécialisée ", Documentation et bibliothèques, avril-juin 1997, p.55 
pancanadien ayant des centres d'information dans plusieurs villes, ceux-ci fonctionnent en réseau sous la supervision d'un directeur national. Les ressources d'information sont également partagées et gérées via un catalogue collectif virtuel partout au Canada.

Les nouvelles technologies de l'information permettent plus facilement cette mise en commun des ressources humaines et physiques. La gestion des technologies de l'information telles que les sites Internet, les portails intranet et la gestion électronique des documents, pour ne citer que ceux-là, est un autre défi que relèvent brillamment les spécialistes de l'information en entreprise. Chez Rio Tinto Alcan, des membres du centre d'information d'affaires ont contribué à la création et à la conception du portail de veille technologique de l'unité d'affaires Métal primaire. Les spécialistes de l'information veillent à sa mise à jour informationnelle quotidienne et agissent aussi en tant que conseillers pour les développements futurs de l'application.

D'ailleurs, ces mêmes technologies de l'information font apparaitre une nouvelle réalité, soit la disparition du centre d'information physique et son remplacement par une bibliothèque virtuelle. Bell Canada est un pionnier dans ce domaine : en fait foi la création du Centre d'information sur les marchés ${ }^{2}$. Certains spécialistes de l'information quittent aussi le giron de la bibliothèque pour s'intégrer à des équipes de travail et ainsi devenir des joueurs importants dans des groupes stratégiques. En conséquence, les compétences des bibliothécaires dans l'identification, l'organisation et la diffusion de l'information sont reconnues à l'extérieur du cadre de la bibliothèque. Citons les agences de recrutement de cadres qui embauchent autant des détenteurs de maitrise en administration des affaires que des spécialistes de l'information comme analystes de recherche.

Finalement, les centres d'information en entreprise sont de plus en plus reconnus comme des services de gestion de ressources d'information stratégique provenant de source interne autant qu'externe. L'entreprise intègre à l'intérieur d'un même service la gestion de la bibliothèque, des documents administratifs et des archives de la société. C'est le cas du Cirque du Soleil, où Louise Guy " (...) supervise a team of six employees that offers research services, develops a physical and electronic library dedicated to the creative teams, and supports activities related to records management, conservation, and archives dissemination ${ }^{3}$."

Bref, le centre d'information en entreprise doit aujourd'hui relever plusieurs défis : fusions et acquisitions, implantation et adaptation aux nouvelles technologies de l'information, gestion des ressources d'information stratégique de l'organisation et remplacement éventuel du centre par un service virtuel.

\footnotetext{
2. Boyd, Stephanie, "What's Next for Corporate Virtual Libraries?», Online, vol. 28 $n^{\circ} 6$, Nov-Dec 2004, p.14.

3. Spencer, Forrest Glenn "It's one big circus», Information Outlook, vol. 11, $n^{0} 10$,
} octobre 2007, p.24.

\section{Le profil de la bibliothèque spécialisée dans les organisations}

Cette section s'attardera à définir et dénombrer les types de bibliothèques spécialisées en entreprises que l'on retrouve au Québec ainsi qu'à décrire leurs principaux services. La norme ISO 2789 définit ainsi les bibliothèques spécialisées :

Une bibliothèque établie et financée par une entreprise, une association privée, une agence ou institution gouvernementale, une organisation à but non lucratif ou un groupe d'intérêt pour répondre aux besoins de ses employés, membres ou personnels en fonction des missions et buts de l'organisme. L'étendue du fonds est alors limitée par les intérêts de l'organisme-hôte 4 .

Pour les besoins de cet article, nous nous sommes limités aux bibliothèques dites d'entreprise, c'est-à-dire celles qui font partie d'une entité à caractère commercial. Sont donc exclues de cette liste les bibliothèques gouvernementales, médicales, d'associations ou d'organismes à but non lucratif ainsi que les fonds d'archives et de recherchetifique et technique).

Nous avons circonscrit cinq types d'entreprise :

- Entreprise : Rio Tinto Alcan, PricewaterhouseCoopers

- Juridique : McCarthy Tétrault, Ogilvy Renault

- Pharmaceutique : Wyeth, Sanofi-Aventis, Novartis

- Paragouvernementale : Caisse de dépôt et placement du Québec, Société des alcools du Québec

- Ingénierie ou recherche en entreprise : SNCLavalin, Bell Helicopter Textron

À l'aide de plusieurs répertoires, nous avons dénombré les bibliothèques spécialisées faisant partie de chaque type d'entreprises ${ }^{5}$ :

$\begin{array}{lc}\text { Types d'entreprise } & \begin{array}{c}\text { Nombre de } \\ \text { bibliothèques } \\ \text { spécialisées }\end{array} \\ \text { Entreprises } & 28 \\ \text { Juridiques (en cabinet) } & 25 \\ \text { Ingénieries/recherches } & 18 \\ \text { Pharmaceutiques } & 10 \\ \text { Paragouvernementales } & 4\end{array}$

4. ISO 2789 (2006), Information et documentation -.. Statistiques internationales de bibliothèques.

5. Ceci inclut :

Répertoire des membres du SLA; Section de l'est du Canada

Répertoire des membres de l'Association des bibliothèques de droit de Montréal Répertoire des membres de l'ASTED

Directory of librairies in Canada.Toronto, ON: Micromedia Ltd., 2006-2007. / Répertoire des bibliothèques du Canada. Toronto, ON: Micromedia Limited, 2006-2007 
Cet inventaire nous a permis de constater que le Québec compte 85 centres d'information spécialisés, principalement situés dans la région de Montréal. Comme les services offerts par ces centres sortent souvent du cadre traditionnel de la bibliothèque, on remarque également une tendance très marquée à ne plus utiliser le terme «bibliothèque " pour se définir. On privilégie maintenant les termes : centre d'information, centre de documentation, centre de recherches informationnelles et documentaires, centre de ressources d'information, services d'information, etc. En effet, les professionnels qui y travaillent offrent de plus en plus de services et d'expertises non traditionnels. En complément aux services de recherches documentaires, ils proposent des services de veille, de conception et de gestion de base de données et de connaissances, de gestion de l'information numérique intégrée, de revues de presse, de collection et de gestion d'information audiovisuelle, d'expertise en taxinomie, de gestion de site Web, etc. Notons aussi que l'archivage et la gestion documentaire sont souvent compris dans les responsabilités des bibliothèques spécialisées (ou centres d'information). Peu importe leur appellation, les bibliothèques gèrent encore très souvent une collection de livres et de périodiques en plus d'offrir les services d'achats, d'abonnements et de prêt entre bibliothèques.

\section{Les services offerts ${ }^{6}$}

Suite à la consultation effectuée auprès de plusieurs professionnels de différents milieux, nous avons constaté l'étendue des services offerts. Les diverses équipes de professionnels de l'information au sein des organisations sont souvent intégrées pour offrir des services de plus en plus rapides et proactifs, en format électronique, en lien avec le développement des ressources intranet et des services de recherches et de veille. En fait, les spécialistes de l'information contribuent au contenu de l'intranet et d'autres portails spécialisés pour disséminer efficacement l'information à leurs clients. De plus, ils travaillent souvent en équipe avec des informaticiens afin de concevoir et de s'assurer de la convivialité et de la facilité d'utilisation (usability) de tels outils au niveau organisationnel. Ces systèmes sont essentiels, non seulement pour gérer des sources externes (par exemple des abonnements, des nouvelles, des fils en format RSS7), mais aussi pour optimiser la gestion des documents produits à l'interne (par exemple des rapports d'analyses). Avec de telles activités surgit le besoin de développer une expertise en négociation de licence d'utilisation multi-usagers pour des ressources documentaires.

6. Cette section a été élaborée grâce à une consultation faite auprès de six centres d'information spécialisés provenant de tous les types de bibliothèques cités précédemment.

7. Selon le Grand dictionnaire terminologique, la définition d'un format RSS est un format propriétaire de syndication de contenu Web, basé sur le XML, qui permet d'indexer de façon automatisée le contenu d'un site Web et de le mettre instantanément à la disposition d'autres sites.

\section{Certains spécialistes de l'information quittent le giron de la bibliothèque pour s'intégrer à des équipes de travail et ainsi devenir des joueurs importants dans des groupes stratégiques.}

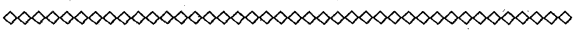

Subséquemment, les bibliothécaires jouent le rôle de conseillers dans l'application du droit d'auteur. La croissance des outils disponibles en ligne pour les usagers oblige aussi les organisations à offrir des formations personnalisées afin de les rendre plus autonomes.

Même si les bibliothèques et les centres d'information en question se trouvent au Québec, leur clientèle devient de plus en plus diversifiée. Les professionnels de l'information québécois appuient des usagers et des équipes spécialisés, impliqués dans des projets variés, partout dans le monde. Il est certain que les besoins d'ingénieurs ou de scientifiques sur le site d'un projet seront plus techniques, tandis qu'au siège social d'une compagnie, ils seront d'avantage concentrés, par exemple, dans les domaines de la gestion ou de la finance. En fait, les professionnels de l'information donnent des services à plusieurs types de travailleurs du savoir (knowledge workers) ou à des gestionnaires. $\grave{A}$ titre d'exemple de clients internes, nous retrouvons des membres de la haute direction, des avocats, des parajuristes, des analystes financiers, des spécialistes en marketing, des spécialistes en développement des affaires, des communicateurs ainsi que des professionnels techniques et scientifiques (ingénieurs, chimistes, médecins, géologues). Enfin, certaines bibliothèques desservent également des clients à l'extérieur de l'organisation.

Mieux connaître les besoins des clients pour fournir des services pertinents, voire personnalisés à l'occasion, pousse les centres d'information à vouloir s'intégrer aux équipes d'affaires de leur organisation. Dans l'avenir, plusieurs centres prévoient offrir encore plus d'outils électroniques (portail, intranet, technologie RSS, services d'alertes, bases de données créées à l'interne) afin de fournir à leurs clients de l'information à valeur ajoutée. Cette nouvelle offre de services électroniques à l'échelle mondiale nécessitera la mise en place de politiques de gouvernance et de meilleures pratiques pouvant encadrer ces initiatives. Les outils de type Web 2.0 ainsi que les réseaux et les médias sociaux joueront sûrement un rôle plus important - mais l'adaptation exacte à ces ressources reste à déterminer.

La promotion de nos services et notre "branding" à l'intérieur de nos organisations demeurent aussi une préoccupation constante dans nos milieux afin d'améliorer la visibilité des centres d'information et de vendre leurs mérites. En effet, la reconnaissance reste toujours pour nous un défi, et plusieurs professionnels de notre 
milieu considèrent ces projets de communication comme une priorité. C'est ainsi que certains planifient déjà des initiatives de marketing à l'interne comprenant des bulletins électroniques ou des activités personnalisées destinées à accrocher des groupes clés.

\section{Les tendances et les enjeux}

Les résultats de l'enquête décrits précédemment illustrent le fait que le monde de l'information est en mutation constante et que les changements s'y opèrent de plus en plus rapidement. Les professionnels se doivent de rester à l'affût des nouvelles tendances et de servir leurs usagers toujours plus rapidement et efficacement. Dans cette optique, il est intéressant de s'interroger sur les tendances, les enjeux et les défis qui guettent le spécialiste de l'information dans les organisations.

Le premier défi vient des usagers eux-mêmes. Les jeunes, et souvent les moins jeunes aussi, sont de plus en plus performants en recherche d'information. Cela entraîne deux conséquences. Premièrement, les questions auxquelles nous répondons sont toujours plus complexes, les recherches de premier niveau pouvant être réalisées par la plupart des utilisateurs. Deuxièmement, il faut convaincre ces usagers que nous pouvons encore être utiles même s'ils ont accès à l'Internet et savent très bien s'en servir. Il faut donc développer des produits et des services à valeur ajoutée permettant notamment à nos clients d'accéder aux couches plus profondes du Web. De plus, la chasse gardée des professionnels a toujours été de connaître et d'accéder à des sources spécialisées et payantes. Par contre, dans nos organisations, beaucoup d'analystes s'abonnent directement aux sources d'information spécialisée dont ils ont besoin. Il faut donc revoir le rôle que nous jouons auprès de nos clients. Si ces derniers sont capables d'utiliser les sources eux-mêmes, le rôle du centre d'information spécialisé devient alors celui de coordonnateur entre les départements et les équipes afin de gérer les abonnements, d'en faire baisser les coûts et d'en assurer le suivi. De plus, les professionnels peuvent toujours soutenir leurs clients qui, heureusement, n'ont pas toujours le temps de tout faire eux-mêmes.

Un autre défi dans les années à venir est la gestion de l'infobésité (surcharge d'information). Il n'a jamais été aussi facile de générer et de trouver de l'information. Toutefois, les professionnels doivent s'assurer de continuer à organiser, classer et retrouver les documents produits par leur propres organisations, tout en visant à ne pas surcharger d'information les usagers auxquels ils répondent. L'intégration des fonctions de recherche, de veille, de gestion documentaire et de gestion de connaissances dans les centres d'information spécialisés est une tendance certaine vers une meilleure gestion du contenu documentaire mais aussi vers une meilleure diffusion de l'information pertinente. Les professionnels dans les organisations semblent très préoccupés par l'implanta- tion de systèmes et de pratiques soutenant la production phénoménale de l'information à l'interne.

Par ailleurs, beaucoup de milieux spécialisés s'intéressent à une nouvelle forme d'information, une information vive, extrêmement difficile à circonscrire, à définir, à classer et à retrouver. Les organisations sont conscientes, plus que jamais, que leur plus grande richesse, leur mine d'information, se trouve avant tout dans la tête de leurs employés. Gérer, conserver, diffuser, réutiliser le savoir est certainement un des défis les plus passionnants et les plus exigeants que les professionnels d'aujourd'hui et de demain auront à relever. Heureusement, le milieu académique offre maintenant des formations spécialisées dans ce domaine. De plus, certaines organisations, par l'entremise de leur centre d'information spécialisé, font déjà office de pionnier dans ce domaine peu exploré et pourront tracer la voie pour d'autres sur le sentier de la connaissance humaine.

\section{Conclusion}

Nous avons tenté dans cet article de démontrer la mutation qui s'opère actuellement dans le milieu des bibliothèques spécialisées au Québec. Nous avons vu que la mondialisation, le développement des technologies de l'information, la naissance de la bibliothèque virtuelle et l'évolution des besoins des utilisateurs obligent les spécialistes de l'information à innover. En effet, le rôle des bibliothécaires, du moins dans les organisations, ne se limite plus au cercle restreint de la bibliothèque traditionnelle. Nous intervenons de plus en plus sur des projets à titre de consultants et de gestionnaires.

Par ailleurs, comment passer sous silence le défi de plusieurs générations de professionnels de l'information, soit celui de faire connaitre notre métier et nos compétences auprès de nos organisations ? Aujourd'hui, ce défi est double : il faut à la fois miser sur nos compétences propres (la recherche, l'organisation, la gestion de l'information) tout en s'assurant de promouvoir la haute employabilité des professionnels de l'information dans des champs encore peu exploités par nos collègues. En effet, il est étonnant qu'à l'ère de l'information, les MSI (détenteurs d'une maîtrise en sciences de l'information) n'aient pas encore obtenu la reconnaissance et le crédit accordés à d'autres professionnels très présents dans les entreprises. Les organisations privées ont tendance à embaucher systématiquement des MBA (détenteurs d'une maitrise en sciences de l'administration) pour des postes où la recherche d'information est prédominante, tels les recherchistes en environnement, en marketing ou en communication, alors que plusieurs de ces postes pourraient être occupés par des diplômés des sciences de l'information. Les bibliothécaires devront en conséquence se concentrer sur la promotion de leur expertise auprès de leurs gestionnaires afin de prendre la place qui leur revient au sein de l'entreprise. (๑) 